எட்டுத்தொகை

நூல்களில்

காற்று குறித்த

சுற்றுச்சூழல்

\title{
விழிப்புணர்வு
}

பி. ரெஜினா அ, *

அ தமிழ்துறை, நிர்மலா மகளிர் கல்லூரி, கோயம்புத்தூர்-641 018, தமிழ்நாடு, இந்தியா.

\section{Environmental Awareness on Air in Eight Anthologies}

\author{
B. Regina ${ }^{a}{ }^{*}$ \\ a Department of Tamil, Nirmala College for Women, Coimbatore - 641 018, Tamil Nadu, India.
}

Corresponding Author: jane.regi@yahoo.com

Received: 27-02-2020 Accepted: 01-04-2020

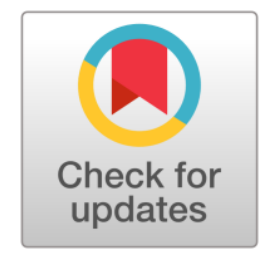

\begin{abstract}
The Sangam literature is proof that the Tamil poets were aware of the nature of the wind. The sweetness and tenderness of the breeze may impress the hearts of the witnesses. The Tamils marked the wind from every direction with each name. It is seen that the wind and summer are blowing fast and the former is a blizzard and the latter is very hot. All the charm of the tenderness is the breeze, the energy and the swiftness of the blow. The winds are explained by the sound of the wind or the whistling of the tree's leaves. Because the anthropogenic man recognized the importance of the air and maintained the airspace clean, there were no significant impacts on humans and other organisms. But, humans, animals, and plants today suffer from many conditions. We, as human beings, recognize the importance of the air, and we have to protect the atmosphere from pollution, especially in situations where man is unable to breathe.
\end{abstract}

Keywords: Sangam literature, Eight Anthologies, Air Pollution

\section{ஆசிரியர் குறிப்பு}

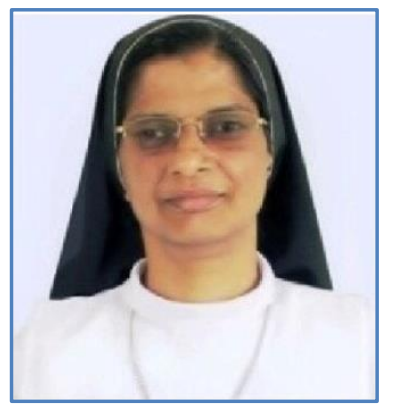

முனைவர் பி. ரெஜினா நிர்மலா மகளிர் கல்லூரியில் தமிழ்த்துறை தலைவராக பணியாற்றி வருகிறார். இவர் ஒரு சிறந்த தமிழ் ஆர்வலராகவும், திறனாய்வாளராகவும் அறியப்படுகிறார். தொடர்ந்து தமிழ் கலை மற்றும் இலக்கிய வெளியில் பயணித்து வரும் இவருக்கு, தமிழ் தொண்டர் விருது, திருவள்ளுவர் விருது, கல்வி செம்மல் விருது, செந்தமிழ் திலகம் விருது, ஆய்வுலக சிற்பி, முத்தமிழ் சுடர் விருது போன்ற பல்வேறு விருதுகள் அளிக்கப்பட்டுள்ளன. "அருளொளி அன்னை தெரசா காவியத்தின் கட்டமைப்பும் மனிதநேய மாண்புகளும்” எனும் தலைப்பில் தனது முனைவர் பட்ட ஆய்வை நிறைவு செய்துள்ளார். மேலும் “எட்டுத்தொகை நூல்களில் இயற்கையும் சுற்று சூழல் பாதுகாப்பும், விழிப்புணர்வும்" எனும் தலைப்பில் பல்கலைக் கழக 
ஆய்வுக்குழுவில் இருந்து ஆராய்ச்சி நிதியாக 1,35,000 பெற்று ஆய்வை நிறைவு செய்துள்ளார். எட்டுத்தொகை நூல்களில் ஆர்வமுள்ள இவர் தொடர்ந்து அத்துறையில் பல ஆய்வுக்கட்டுரைகளை எழுதிவருகிறார். பல்கலைக் கழக மானியக்குழுவால் அங்கீகரிக்கப்பட்ட ஆய்விதழ்களில் இவருடைய கட்டுரைகள் தொடர்ந்து வெளிவந்து கொண்டிருக்கின்றன.

\section{ஆய்வுச் சுருக்கம்}

சங்கப்புலவர்கள் காற்றின் தன்மைகளை மிக நுட்பமாக அறிந்திருந்தனர் என்பதற்கு சான்றாக சங்க இலக்கிய பாடல்கள் விளங்குகின்றன. தென்றலின் இனிமையும் மென்மையும் சங்கத்துச் சான்றோர்களின் இதயத்தைக் கவர்ந்தன என்பதை உணரலாம். ஒவ்வொரு திசையிலிருந்தும் வரும் காற்றினை ஒவ்வொரு பெயரால் தமிழர்கள் குறித்தனர். வாடையும் கோடையும் வேகமாக வீசுகின்றவை என்பதையும், முன்னது பனிப்புடையதாகவும் பின்னது பெருவெப்பம் உடையதாகவும் வீசுவன என்பதையும் உணரலாம். மென்மையின் கவர்ச்சியெல்லாம் பெற்றது தென்றல், ஆற்றலும் விரைவும் இல்லாமல் வீசுவது கொண்டல். இந்நான்கு காற்றுகளும் மரம் செடிகளின் இலை மலர் காய் கனிகளை அசைப்பதாலோ உதிர்ப்பதாலோ எழும் ஓசைகள் வாயிலாக விளக்கப்படுகின்றன. சங்ககால மனிதன் காற்றின் முக்கியத்துவத்தை உணர்ந்து காற்று மண்டலம் மாசுறாத வண்ணம் பாதுகாத்து பராமரித்து வந்ததால் மனிதனுக்கும் மற்ற உயிரினங்களுக்கும் எவ்விதத்திலும் பெரிய பாதிப்புக்கள் ஏற்படவில்லை. இன்று மனிதர்களும், விலங்குகளும், தாவரங்களும் பல நிலைகளில் பாதிக்கப்படுகின்றன. குறிப்பாக மனிதன் சுவாசிக்க தூயகாற்று கிடைக்காத சூழ்நிலையில் பல்வேறு சுவாச நோய்களுக்கு ஆளாகி மடியக்கூடிய நிலை உருவாகி வருவதால் மனிதர்களாகிய நாம் காற்றின் முக்கியத்துவத்தை உணர்ந்து காற்றை மாசுறாத வண்ணம் பேணிக் காப்பது நம் ஒவ்வொருவருடைய கடமையாகும்.

\section{முன்னுரை}

தமிழ் மக்கள் காற்றின் போக்கினைத் தெளிவாக உணர்ந்திருந்தன என்பதற்கு சான்றாக சங்கப் பாடல்கள் அமைந்துள்ளன. இயற்கை நமக்களித்துள்ள விலை மதிப்பற்ற செல்வம் காற்றாகும். உலகிலுள்ள அனைத்து உயிரினங்களும் உயிர் வாழ அடிப்படைத் தேவையாக காற்று உள்ளது. பூமியைச் சுற்றியுள்ள காற்று மண்டலம் பூமிக்கு ஒரு பாதுகாப்பு வளையமாகத் திகழ்கிறது. எல்லோராலும் வெறுக்கப்படுவது வடக்கேயிருந்து வரும் வாடைக்காற்றாகும். வாடையின் குளிர்க்கொடுமை இரவிலே பொறுக்க முடியாத அளவு பெருகுகிறது. நாரைகள் குளிர் தாங்க மாட்டாமல் துயரம் மிகுந்த குரல் எழுப்புவதும், நண்டுகள் வாடைக் குளிரால் வாடித் தம் மண் வளையுள் ஒடுங்குவதும் புலவர்களால் குறிக்கப்பட்டுள்ளன.

கிழக்குத் திசைக்காற்றைப் பற்றிய குறிப்புக்கள் பெரும்பாலும் கடற்கரையை வருணிக்கும் போதே வருகின்றன. கடற்கரையில் உள்ள மணல்மேடு கொண்டல் என்னும் 
கீழ்த்திசைக் காற்றால் எழுப்பப்படுவதாகப் புலவர்கள் குறிக்கின்றனர். கொண்டல் அசைவளி முதலான தொடர்களிலிருந்து கொண்டலாகிய கீழ்த்திசைக்காற்று மெல்லென வீசும் இயல்பினது என்பதை அறியலாம். கொண்டல் தென்றல் ஆகியவற்றைவிடக் கோடையாகிய மேலைத்திசைக்காற்று அடிக்கடிப் புலவர்களால் குறிக்கப்பட்டுள்ளது. சங்க இலக்கிய மரபிலே வாடைக்கு அடுத்த நிலையில் கோடை இடம் பெறுவதாகச் சொல்லலாம். கோடை முதுவேனிற் பருவத்து வீசுவதாகையினால், கோடையின் வெம்மை மிகுதியாகவே இருக்கும். தென்றலின் இனிமையும் மென்மையும் சங்கத்துச் சான்றோர்களின் இதயத்தைக் கவர்ந்தன என்பதை உணரலாம். தென்றல் இளவேனிற் பருவத்தினது என்பதைச் சங்கஇலக்கிய மரபு புலப்படுத்துகின்றது (Balasubramaniyan, 2007; Nagarajan, 2007; Dhatchenamoorthi, 2007; Alis, 2007; Subramaniyan and Balasubramaniyan, 2007; Viswanathan, 2007; Jeyabal, 2007; Balasubramaniyan et al., 2007)

ஒவ்வொரு திசையிலிருந்தும் வரும் காற்றினை ஒவ்வொரு பெயரால் தமிழர்கள் குறித்தனர். வாடையும் கோடையும் வேகமாக வீசுகின்றவை என்பதையும், முன்னது பனிப்புடையதாகவும் பின்னது பெருவெப்பம் உடையதாகவும் வீசுவன என்பதையும் உணரலாம். மென்மையின் கவர்ச்சியெல்லாம் பெற்றது தென்றல், ஆற்றலும் விரைவும் இல்லாமல் வீசுவது கொண்டல். இந்நான்கு காற்றுகளும் மரம் செடிகளின் இலை மலர் காய் கனிகளை அசைப்பதாலோ உதிர்ப்பதாலோ எழும் ஓசைகள் வாயிலாக விளக்கப்படுகின்றன. கொன்றையின் இனிய சுவையையுடைய கனிகள் பாணர் தம் பறையை முழக்கும் குறுந்தடிகளோ என்று ஐயுறுமாறு கொடிய காற்று வீசீயதால். பாறையில் விழுகின்ற வண்ணம் கிளைகள் துவண்டு (நற். 46/6-8) ஆடியது. குறுகிய உயரத்துடன் நிற்கும் குரவமரத்தில் சிறிய அரும்புகளுடன் மலர்களும் நன்கு மலர்ந்து நின்ற மலர்களின் மணத்துடன் தென்றல் காற்று கலந்து வீசீயது (நற். 56/1-2) என்கிறார்.

வேர்கள் ஒன்றோடொன்று பிணிப்புற்றிருக்கும் மூங்கில்களில் காற்று மோதியதால் உண்டான ஓசையானது தறியிலே கட்டப்பட்ட யானை வருந்திப் பெருமூச்சு விட்டது போன்றிருந்தது (நற். 62/1-2) என்கிறது. மலர்கள் அவிழ்கின்ற புதிய மணல் பரந்த கடற்கரைச் சோலையில் உள்ள புன்னையின் நுண்ணிய மகரந்தப் பொடியானது வீசுகின்ற கீழைக்காற்று வந்து மோதிய போதெல்லாம் குருகின் வெண்மையான முதுகில் நிரம்பக் குவித்த தன்மை இங்கு விளக்கப்பட்டுள்ளது (நற். 74/6-9) எனவும், கார் காலத்தின் மிக்க முன்பனிப்பருவத்தில் மயிர்களைக் கொண்ட காய்களையுடைய உழுந்தின் அகன்ற இலைகள் எல்லாம் நீங்கும் படியாக வீசி நம்மை விட்டு நீங்காமல் நாள்தோறும் வருத்துகின்ற அன்பு செய்யாத வாடைக்காற்று வீசியது (நற். 89/5-7) எனவும், மூங்கில் அடர்ந்த காட்டுப்பகுதியிடத்து வீசுகின்ற காற்று வெம்மையான காய்ந்த கொடிகள் சுற்றி விளங்குவதும் முட்கள் நிறைந்த அடிப்பகுதியுடையதுமான இலவ மரத்தின் கிளைகளை அதிரச் செய்வதோடன்றி அவற்றை முறியுமாறும் செய்தது (நற். 105/1-3) எனவும் காற்றின் தன்மைகள் சொல்லப்பட்டுள்ளன.

$$
\text { . இணர்ப் பாலை, }
$$

செல் வளி தூக்கலின், இலை தீர் நெற்றம் கல் இழி அருவியின் ஒல்லென ஒலிக்கும் 
புல் இலை ஒமைய" (நற். 107/3-6)

என்ற பாடல் வழியாக வெண்பாலை மரம் இயங்கும் காற்றினால் இலை பலவும் உதிர்ந்த கிளைகளில் காய்ந்த நெற்றுக்கள் மலையினின்று வீழும் அருவி போல ஒல்லென ஓலிக்கும். ஓமை மரம் புல்லிய இலைகள் கொண்டு விளங்கியதை அறியமுடிகிறது.

வேனிற் காலத்தில் இத்தி மரத்தின் நெடிய விழுது நிலத்தில் தோயாது தொங்க அதன் மீது நாராற் பிணித்த ஊசலைப் போல மேல் காற்று வீசிய பொழுதெல்லாம் அவ்விழுது ஆடியது (நற். 162/9-10) என்பது தெளிவாகிறது. நம் தலைவர் சுழன்று அடிக்கின்ற சூறைக்காற்றை உடையதுமான சுரத்திடத்தே (நற். 189/10) சென்றார். தலைவனின் மலையில் உயர்ந்த நெடிய சிகரத்திலே பரவிக், குளிர்ச்சியுடன் அகன்ற பாறையில் காற்று அளாவி (நற். 236/7-9) வீசியது எனக் குளிர்ந்த காற்றின் தன்மை, மெல்லிய நுண் மணல் தோன்றும் மென்மையுடன் வீசும் வாடைக்காற்று மிகுதியாய் மோதித் தீண்டியது (நற். 241/3-4) என வாடைக்காற்றின் இயல்பு, ஆகியன தெரிய வருகின்றன.

கரிய புனத்திலுள்ள கருங்காக்கணத்தின் கண் போன்று மலர்ந்த கரிய மலர் வாடைக்காற்று வீசியதால், ஆடுகின்ற மயிலின் தோகை அசைவது போல (நற். 262/1-2) அசைந்தாடியது. செறிந்த கட்டவிழ்ந்து மலர்ந்த தாழம் பூக்கள் மிகுந்துள்ள பெரிய புதரில், வீசுகின்ற மேல்காற்று மாதுவதால் மலரின் நுண்ணிய தாதுக்கள் எல்லா இடங்களிலும் பரவியமை (நற். 299/2-3) தாழை சோற்றைச் சொரிகின்ற குடை போலக் கூம்பிய அரும்பினை மலரச் செய்த. காற்று, அப்பூமடலுட் புகுந்து பரவி வீசியதால் குன்றாத நல்ல மணத்துடன் விளங்கியமை (நற். 335/4-6) ஆகியன தெளிவாகின்றன.

“கானற் கண்டல் கழன்று உரு பைங் காய்

நீர் நிற இருங் கழி உட்பட வீழ்ந்தென” (நற். 345/1-2)

என்ற பாடல் வாயிலாக காற்று வீசுவதால் கடற்கரைச் சோலையிலமைந்த கண்டல் மரத்திலிருந்து பசிய காய்கள் கழன்று உதிரும். அக்காய்கள், நீல நிறமுடைய பெரிய கழியிடத்தே உள்ளே செல்லுமாறு வீழும் என்பதைப் பற்றி அறியமுடிகிறது.

உப்பங்கழிகளில் உள்ள சோலைகளில் உள்ள பனை மரங்கள் காற்றாலசைக்கப்பட்டு ஓலைகளை வீழ்த்தியது (நற். 354/1-2) என்கிறது. பகலிலும் இரவிலும் இடியுடன் கூடிய மழை பொழிதலால் குளிர்ந்த வாடைக் காற்றுடன் பனி பொழிந்தது (நற். 364/2-3) என்கிறது. மேற்கண்ட பாடல்களின் வழியாக காற்று பற்றிய செய்திகள் தெரிய வருகிறது.

மேல்காற்று வீசும்போது நிலை கலங்கி, வாகை மரத்தின் வெண்ணிற நெற்றுகள் ஒலி (குறுந். 7/4-5) எழுப்பியது. சுழன்று அசைந்து வீசும் தென்றல் காற்று தலைவியை வருத்தியதாக (குறுந். 28/4) கூறுகின்றாள். கரும்பினது குவிந்த அரும்புகள் மலரும்படி, நுண்ணிய மழை பொழிந்து, பின்னர் சிறுசிறு துளியாகப் பெய்கின்ற குளிர்ச்சியாக வீசும் வாடைக் காற்றினையுடைய கூதிர்காலம் (குறுந். 35/3-5) ஆகும். மேலும் வெம்மையான, வலிமையுடைய விரைவான காற்று, மரக்கிளைகளில் வீசுவதால், வாகை மரத்தின், நெற்றுக்களின் முற்றிய வற்றல்கள் ஒலித்தற்கு இடமாக உள்ள மலைகளையுடைய சுரமாகும் (குறுந். 39/1-3) என்றும் சொல்லப்பட்டுள்ளது.

“கூன் முள் முண்டகக் கூர்ம்பனி மாமலர் 
நூல்அறு முத்தின் காலொடு பாறித்

துறைதொறும் பரக்கும் தூமணற் சேர்ப்பனை" (குறுந். 51/1-3)

வளைந்த முள்ளையுடைய முண்டகத்தின் மலர் பனிக்காலத்தில் கோத்தநூல் அற்ற முத்துக்களைப் போல, காற்றினால் சிதறி வீழ்ந்து, நீர்த்துறைகள் தோறும் தூயமணற்பரப்பினை அழகுடையதாக்கியது. குறுகிய தாளினையுடைய கூதளங்கொடி, காற்றில் நிலையின்றி அசைவதால், அதனைப் பற்றிக் கொண்டு முடவன் மலையில் ஏற அதற்குரிய வலியின்மை (குறுந். 60/1-2) 2ணர்த்தப்பட்டது. குளிர்ந்த வாடைக்காற்று, மலைப்பக்கத்தில் உள்ள சேம்பின் அசைதலையுடைய வளம்பொருந்திய இலையைப் பெரிய களிற்றின் செவியைப் போலத் தோன்றும்படித் தடவி அசைத்தது (குறுந். 76:4-5) என்கிறது.

நீரில் உள்ள, மலரும் செவ்வியையுடைய அரும்பினை மலரச்செய்து, புதர்களில் உள்ள மயில்பீலியின் ஒள்ளிய கண்களைப் போன்ற கருவிளை மலர்களை அசைத்து, நுண்ணிய முள்ளையுடைய ஈங்கையின் செந்நிறமுடைய அரும்புகளின் நிறத்தையும், மென்மையும் உடைய மலர்களை உதிர்த்து, குளிர்ச்சியுடையதாகித் துன்பத்தைத் தரக்கூடிய வகையில் வாடைக் காற்று வீசியது (குறுந். 110/2-7) எனவும், இருள் செறிந்த யாமப்பொழுதினையுடைய மிக்க குளிர்ச்சியுடன் வாடைக் காற்று வீசியது (குறுந். 160/4-5) எனவும், கீழ்க்காற்றால் வீழ்த்தப்பட்ட ஒள்ளிய சிவந்த காந்தள்மலர்கள் பாறையின் மேல் கவிழ்ந்து வீீழ்து கிடந்தது (குறுந். 185/6) எனவும், காட்டில் வளர்ந்த இருப்பை மரத்தில் வேனிற் காலத்தில் வரும் வெள்ளிய பூக்கள், நிறைந்த நெடிய கிளைகளில் காற்று மோதியது (குறுந். 329/1-2) எனவும், பாலை நிலத்தில் அருவழிகளில் உள்ள வாகை மரத்தின் நெருங்கிய வெள்ளி நெற்றுக்களின் விதைகள், உள்ளே இடப்பட்ட சிலம்பின் பரல் ஒலிப்பதைப் போல, மேல்காற்று வீசுயதால் ஒலித்தது (குறுந். 369/1-2) எனவும், கடிய காற்றுவீசித் தொகுத்த மணற்குவியலாக இருந்தது (குறுந். 372/2) எனவும் காற்றின் தன்மைகள் தெரிய வருகின்றன.

குவளை

கோடை ஒற்றினும் வாடா தாகும்" (குறுந். 388:1-2)

குவளை மலர், மேல் காற்று மோதிய போதினும், வாடாது வளர்ந்தது எனவும் சொல்லப்பட்டுள்ளன.

மாவினையுடைய உயர்ந்த கொம்புகளினின்றும் மிக்க காற்றால் அசைக்கும் நறிய வடுக்கள் இருந்தன (கலி. 84/1) என்கிறது.

“........ கடிகாவில் கால் ஒற்ற, ஒல்கி ஒசியா” (கலி. 92/51-52)

மணம் மிக்க மலர்க்காவிலே, காற்றடிக்க, கொடிகள் ஓங்கி வளர்ந்து ஒடியாது விளங்கின.

காற்றைப் போலே விரைந்து வந்த சினமுடைய ஏறாகிய காரியைப் பலரும் வந்து சேர்ந்தனர் (கலி.103/40) என்கிறது. நிறைந்த இராக்காலத்தே அசைந்து வருகின்ற காற்றுத் தான் துணையாகி வருத்தாது (கலி. 121/12) நின்றது. பூக்கள் மலரும் செவ்வி இடத்து அசைந்து காற்று (கலித். 126/12) வந்து தன் மேல்பட்டது. வாடைக்காற்று அசைத்தலினால் வளைந்த தாழையின் அசைகின்ற கொம்பில், அசைந்து, நடக்கின்ற நடையினை உடைய நாரை (கலி. 128/2-3) அமர்ந்திருந்தது. 
"மா மலர் முண்டகம் தில்லையோடு ஒருங்கு உடன்

கானல் அணிந்த உயர்;மணல் எக்கர் மேல்” (கலி .133/1-2)

என்ற பாடல் கரிய மலர்களையுடைய கழிமுள்ளி, தில்லையோடு ஒரு சேர, கானலிடத்து, காற்றால் உயர்ந்த இடுமணல் மேல் அழகுடன் விளங்கியது என்று காற்றின் வகைகள், செயல்கள் கூறப்பட்டுள்ளன.

பசிய புதர்களிடையே ஓங்கி வளர்ந்த வேழத்தின் வெண்மலர்கள் காற்றால் அசைந்து வான்வெளியில் (ஐங். 18/1-2) பறந்தது என்றும், நாணல் கரிய நிறமுடைய தண்டான் கோரையை ஒத்த செருந்தியோடு கரும்பினைப் போலக் காற்றால் அலைப்புண்டு சுழலுதற்கு இடமான ஊர் (ஐங். 18/1-2) என்றும் கூறப்பட்டுள்ளன.

மடப்பம் பொருந்திய நடையைக் கொண்ட நாரை, தன் அலகினால் கோதிய பொழுது உதிர்ந்த இ இறககளைக் காற்றால் குவித்த மணற்குன்றுகள் (ஐங். 153/2-4) தோன்றின. நீலமணி போலும் நெடிய மலை, காற்றால் மலரும் அவரைப்பூ போலும் நிறமுடைய வெண்ணிற முகட்டினையும், கரிய உடலையும் உடைய மழை முகிலைச் சூடிக் கொண்டு இடைவிடாது என் கண்முன்னே தோன்றுகின்றது (ஐங். 209/3-5) என்கிறது. வீசும் தண்ணிய, நடுக்கத்தைத் தரும் வாடைக் காற்று வீசுதற்கு உரிய பனிக்காலம் வருவதற்கு முன்பே (ஐங். 223/4-5) நம் காதலர் வந்துவிட்டார் எனவும், குளிர்ச்சி மிக்க வாடைக்காற்றோ மிகக் கொடியதாய் வீசியது (ஐங். 233/1) என்றும், எங்கள் வளம் மிகுந்த இனிய நெடிய இல்லத்தில் யாம் இருப்பவும், அ/தும் தனிமைப்பட்டு இன்னாதாகும் வகையில் வாடைக்காற்றும் எம்மை அலைக்கின்றது (ஐங். 236/1-3) என்றும், விரைந்து பெய்யும் தாங்குதற்கு அரிய பனியொடு கலந்த, கூதிர்ப்பருவத்து வீசும் இயல்புடைய கடுங்குளிருடைய வாடைக்காற்று வருவதற்கு முன்னே வந்தான் (ஐங். 252/3-5) என்றும் கூறப்பட்டுள்ளன.

“வேனில் அரையத்து இலை ஒலி வெரீ இ,

போகில் புகா உண்ணாது, பிறிது புலம் படரும்

வெம்பு அலை அருஞ் சுரம்” (ஐங். 325/1-3)

என்ற பாடலில் கோடைக்காலத்து வெப்பக் காற்றால் அலைப்புண்டு அலைய மரத்தின்; இலைகள் எழுப்பும் ஓசையைக் கேட்டு அச்சமுற்ற போகில் என்னும் புள், இரைதேடி உண்டலைக் கைவிட்டு, வேறு புலங்களை நாடிப்பறத்தற்கு இடனான, கோடை வெப்பம் அலைக்கின்ற கடத்தற்கரிய சுரமாகும் என்ற செய்தி தெரிய வருகிறது.

நுண்மையும் கருமையும் கொண்ட மணல் தென்றல் காற்று வீசுவதனால் வரி வரியாக நுடங்கித் தோன்றுதற்கு இடமானதுமான இளவேனிற் பருவம் (ஐங். 341/3) வந்துவிட்டது. நெருங்கித் தழைத்த இலைகளையுடைய வாழையின் பருத்த அடிப்பகுதி அசையுமாறு தாங்குதற்கு அரிய வாடையும் சுழன்று (ஐங். 460/3-4) வீசுகின்றது. இப்பெரிய நிலம் முழுவதும் குளிருமாறு காற்றை வீசச்செய்து, அதனுடன் யாராலும் பொறுத்துக் கொள்ள முடியாத பனியை நாள்தோறும் (ஐங். 470/1-2) கலக்கச்செய்தது எனக்காற்றின் தன்மைகள் எடுத்துரைக்கப்பட்டுள்ளன.

கடலிலே மலைபோல அலைகள் பெரியனவாய் எழுகின்றன. அவ்வலைகள் சிறுசிறு துளிகளாக உடைந்து சிதறும் வகையில் காற்று பாய்ந்து அடிக்கிறது. எனவே கடல் 
அசைகின்ற நிறைந்த நீரினைச் சூல்போலக் கொண்டு (பதிற். 11/1-2) விளங்குகின்றது. காற்றானது மிக்க வேகத்துடன் சுழன்று அடிப்பதற்கு ஏற்ற வகையிலான நல்ல பெரிய பரப்பினைக் கொண்டிருந்தது. (பதிற். 17/11) என்ற செய்தி தெரிய வருகிறது. மேகங்கள் படிந்து கடலின் நீரைக் கொள்ளுதலால் குறையாமல் ஆற்று நீரும் தன்னிடத்தே வந்து புகுதலால் நிறைந்து கரைக் கடவாமலும், குறுக்காக வீசும் காற்றுச் செலுத்தலால், அசைதலையுடைய மிகவும் நிறைந்த நீரினையுடையதாக (பதிற். 45/19-20) கடல் விளங்கியது.

“வரை மிசை இழிதரும் அருவியின், மாடத்து

வளி முனை அவிர்வரும் கொடி நுடங்கு தெருவில்” (பதிற். 47/3-4)

என்ற பாடல் மலையின் மேலிருந்து வீழ்கின்ற அருவியைப் போல மாடத்தில் காற்றினிடத்தே விளங்குகின்ற கொடிகள் அசைகின்ற தெருவினையுடைய நகராக விளங்கியது எனக்குறிப்பிடுகிறது.

நீரையடைந்த கரையில் நண்டுகள் விளையாடியதனால் உண்டாகிய சுவடுகளை மறைக்கின்ற நுண்ணிய மணலைக் காற்று வீசிகின்ற தூய கரிய பனஞ்சோலையில் அலங்காரத்தாற் பொலிவு பெற்றது (பதிற். 51/7-9) என விளக்குகிறது. பலாமரத்திற் பழுத்த பசியப் புண்பட்ட வாய்போல் வெடித்த பழத்திலிருந்து அரித்து விழுகின்ற தேனை, காற்றானது வீசுகின்ற பறம்புநாடு பொருந்தி பெருவிறலையுடையவன்" (பதிற். 61/1-2) என்ற செய்தியை அறியமுடிகிறது.

திருமாலின் மார்பில் அணிந்த மாலைகளின் மணம் காற்றின் வேகத்தில் கலந்து பரவியது.

(பரி. 1/24) எனத் தெரிவிக்கிறது. சீறிவரும் சூறாவளிக்காற்றைப் போன்று உன்னுடன் போரிடுவதற்கு எதிர் வந்து நின்ற அவுணர்களுடைய கொடிகள் அற்று முறிந்து வீழும் படித்திருமாலின் சங்கு (பரி. 2/37-38) முழங்கியதையும், தடையில்லாமல் செல்லக்கூடியதும் தூய்மை-யுடையதும் உருவம் அற்றதுமாகிய தென்றல் காற்றைப் பாராட்டும் வகையில் திருப்பரங்குன்றம் அமைந்திருந்ததையும் (பரி. 6/53) அறியமுடிகிறது. “வளி வாங்கு சினைய மாமரம் வேர் கீண்டு” (பரி. 7/14) என்ற பாடல் காற்றினால் அலைக்கப்பட்டு முறிந்த கிளைகளையுடைய பெரிய மரங்கள் வேருடன் பறித்துத் தள்ளியது. உனது திருப்பரங்குன்றத்திலிருந்து மதுரைக்குச் செல்லும் வழி. பல்வேறு வகையான மணத்தையும் தழுவிக்கொண்டு தென்றல் காற்று தவழ்ந்து செல்லும் தலைமையுடையது (பரி. 8/26-28) எனவும்

“மன்றல் கலந்த மணி முரசின் ஆர்ப்பு எழ,

காலொடு மயங்கிய கலிழ் கடலென" (பரி. 8/30-31)

எனும் பாடல் வழி மதுரை மாநகரில் மணம் பொருந்திய, மணி போன்ற நிறமுடைய முரசத்தின் முழக்கம் காற்றினால் மோதி அடிக்கப்பட்ட கடல் அலைகள் கரைக்குப் பெயர்ந்து வந்து ஒலிப்பது போல ஒலித்தது எனவும் அறியமுடிகிறது.

மதுரை நகரத்திலுள்ள அழகிய மேல்நிலை மாடங்களின் உள்ளிருந்து மகளிர் தூவிய பனிநீர் மணத்தோடு கலந்து தென்றல் காற்று மணமுடையதாய் (பரி.10/122-123) மாறியமையும், 
நெகிழ்ந்த இதழ்களையுடைய ஒளிமிக்க நீலம் காற்றினால் மலரப் பெற்றமையும் (பரி. 11/22) இயம்பப்பட்டுள்ளன.

“ஓரு திறம், பாடல் நல் விறலியர் ஒல்குபு நுடங்க,

ஒரு திறம் வாடை உளர்வயின் பூங் கொடி நுடங்க" (பரி. 17/15-16)

என்ற பாடல் ஒருபுறம் பாடுமியல்புடைய நல்ல ஆடல் மகளிர் அசைந்தாட, மறுபுறம் காற்று அசைத்ததால் பூங்கொடி மென்மையாய் அசைந்தது எனக் குறிப்பிட்டுள்ளது.

வண்டுகள் மொய்க்கும் மாலைகளைப் பூண்ட, மலைபோலுயர்ந்த தோள்களைக் கொண்ட மைந்தர் தம் மார்பிற் பூசிய சந்தனக் குழம்பிலே படிந்து வந்து வீசும் காற்று, கயல்மீன் போலும் கன்னியராகிய மகளிர் தம் பூந்தாது உதிர்க்கப்பட்ட முகில் போலும் கூந்தலினூடே புகுந்து அசைத்து வரும் காற்று, தேர் உருள் போன்ற வடிவு கொண்ட மலர்களின் கொத்துக்கள் செறிந்த கடப்ப மரத்தின்கண் பொருந்திய நெடுவேளாகிய நினக்கு அன்பர்கள் நிகழ்த்தும் பூசைக் கண்ணே, தூபமிடும் புகையூடே புகுந்து வெளிப்படும் காற்று ஆகிய பலவகைக் காற்றும், நன் திருப்பரங்குன்றத்தில் எப்பொழுதும் (பரி. 21/46-51) வீசிக்கொண்டிருக்கிறது. துடியின் தாள கதிக்கு இசைய ஆடல் மகளிர் கூத்து நிகழ்த்த, அக்கூத்திற்கு மாறாகக் காற்று என்னும் நட்டுவாணன் மெல்லிய பூங்கொத்துக்களைக் கொண்ட கொடியைக் கண்டோர்க்கு இன்பம் மிகுமாறு ஆடச் செய்தான் (பரி. 22/42-43) என்றும், மேருமலையினைப் பெயர்த்து விடுவேன் என்று மிக்க வலிமையுடன் வந்து மோதிய காற்று தேவனுடைய மேலான வலிமையெல்லாம் தம்மைக் கடந்து அம்மலையில் புகுந்து தாக்காதவாறு, அக்காற்றிற்கு மாறாக அம்மலையினைச் சுற்றிக் காத்தவர் ஆதிசேடனாரேயாவார் (பரி.தி. 1/76-77) என்றும் இயம்புகிறது.

இருப்பை மரங்களில் மொட்டுக்கள் அரும்பின; செப்புத்தகடுகள் போன் அம்மரங்களின் சிவந்த தளிர்களின் இடம் எல்லாம் நெய்யை ஒத்த இனிய துளையுள்ள பூக்கள் தமது ஆர்க்குகள் கழன்று, காம்பினை நீக்கிக் காணத்தக்க துளையினையுடையவாய் வானிலிருந்து வீழுகின்ற பனிக்கட்டி போலக் காற்றால் சிதறுண்டு (அகம். 9/3-7) விழுந்தன. கீழ்க்காற்றினால் நிறம் மிக்க கடல் அலைகள் மணல் மேட்டை (அகம். 10/8-9) உடைத்தது. நீண்ட அடியினையுடைய இலவமரங்களில் மலர்ந்த புதிய பல மரங்கள் காற்று வீசுவதால், விழாக்களைத் தன்னிடத்தே கொண்ட வெற்றியையுடைய பழைய ஊரில் நெய் ஊற்றப் பெற்ற விளக்கினின்றும் வீழுகின்ற சுடர்போன்று உதிர்ந்து (அகம். 17/18-20) விழுந்தது. கீழ்க்காற்று கொண்டு வந்து குவித்த மணலில் குரவைக் கூத்தாடினோம் (அகம் 20/7) என்றும், வல்லான் ஒருவன் பெருங் கொம்பினை அடித்து அசைக்கும் கோல் போலத் தென்றல் காற்று மராமரத்தை மலர்கள் உதிர்மாறு தாக்கி அலைத்தது (அகம். 21/10-12) என்றும், நீண்ட அடிப்பகுதியினைக் கொண்ட யாமரத்தின் பொலிவற்ற உயர்ந்த கிளைகளில் இரைச்சலுடன் காற்று ஊடறுத்துச் சென்றது (அகம். 51/2-3) என்றும் இயம்பபட்டுள்ளது.

"குறுங் கால் இற்றிப் புன் தலை நெடு வீழ்

இரும் பிணர்த் துறுகல் தீண்டி, வலி பொர,

பெருங் கை யானை நிவப்பின் தூங்கும்"

(அகம். 57/6-8) 
என்ற பாடல் மூலம் குறிய அடியையுமுடைய இற்றி மரத்தின் புல்லிய உச்சியினைக் கொண்ட நீண்ட விழுது பெரிய சுரசுரப்பினையுடைய உருண்டைக் கல்லைத் தீண்டிக் காற்று அடித்தலால் அசைந்தாடுவது, பெருங்கை யானை தன் கையை உயர்த்தி அசைப்பது போல் தோற்றம் அளித்ததாகத் தெரிவிக்கிறது.

குறவரின் முற்றத்தில் மூங்கிலின் அழகிய பூக்கள் உதிரவும், நீண்ட இதழ்கள் கொண்ட காந்தள் பூவின் நெடிய மடல் ஒடியவும், பனி தோன்றும் முன்பனிக் காலத்தில் வாடைக்காற்று (அகம். 78/7-10) குளிர்ந்து வீசியது. அசைந்தாடும் மூங்கிலில் வண்டுகளால் துளைக்கப்பெற்ற துளையிடத்தே, மேல் காற்றினால் எழுகின்ற அழகிய ஒலி குழல் இசையாக (அகம் 82/1-12) முழங்கின. ஆம்பல் இலையை, அசைத்து வரும் வாடைக் காற்று விட்டு விட்டுப் புகுந்து அலைத்தலால், அவ்விலை ஊதப்பெறும் கொல்லனது உலைக்களத்து விசை கொண்டு இழுக்கும் துருத்தியைப் போலப் புடைத்து (அகம். 96/6-7) சுருங்கிருந்தது. சுழன்று வரும் மேல் காற்றினால், புல்லிய அடிமரத்தினையுடைய முருங்கையின் முதிர்ந்து வீழும் பல பூக்கள், குளிர்ந்த கார்காலத்து ஆலங்கட்டி போலப் பரந்து உதிர்ந்து (அகம். 101/1416) கிடந்தது.

ஓடை என்னும் பெயர் கொண்ட குன்றத்தின்கண் உலர்ந்த தலையினையுடைய ‘ஞமை’ என்னும் மரத்தின் மீது பின்னிய சிலந்திக் கூடானது மேல் காற்றினால் அசைந்து ஆடியது (அகம். 111/5-6) எனவும், வழிப்போவார்க்குத் துன்பத்தைச் செய்யும் மழவரது வண்டிச் சக்கரம் கிழித்து உண்டாக்கிய சுவடான வழியில் வணிகர் கூட்டத்தார் செல்வர்; அவர்கள், தாம் சோறு கட்டிக் கொண்டு வந்து உண்டு எறிந்த வெண்மையான பனை ஓலையால் முடையப்பட்ட குடையினை, அம்பு போலும் வேகத்தைக் கொண்ட சூறாவளிக்காற்று தூக்கி வீசி எறிந்தது (அகம். 121/11-13) எனவும், வாடிய பல அகன்ற இலைகள், ஒல்லென்னும் ஒலியுண்டாக மேல் காற்றினால் உதிர்க்கப் பெற்று அ அடித்துச் செல்லப்பட்டன (அகம். 143/3-4) எனவும், இடமெங்கும் பரவி மிக்க காற்று வீசுந்தோறும் கலங்கியதும், புள்ளிகளையும், வரிகளையும் கொண்ட கலைமானின் தலையின்கண் புதிதாக முளைத்துக் கிளைத்த கொம்பு போன்ற சுவடுகளையும், குறிய காலினையும் உடைய வாகை மரத்தினது கிளையில் விளைந்த நெற்றின் குலை, ஆடும் கூத்தியர் அடிக்க ஒலியுண்டாக்கும் பறை போல, கேட்பார் வியக்கும் வண்ணம் ஒலியுண்டாக்கியது (அகம். 151/5-10) எனவும் குறிப்பிடப்பட்டுள்ளது.

வேனிற் காலத்துச் சுடுகின்ற கதிரவனின் வெம்மை காய்ந்து கெடுத்த கானகத்தில், வீசும் பெருங்காற்று, தழைத்த மூங்கிலின் கணுக்களைப் பொருந்தித் தாக்கும். காற்று அடிக்கையினாலே காம்பினின்றும் கழன்று வீழும் தேன் மணக்கும் புதுமலர்கள், கையினால் விடப்பெறும் சுடர்ப்பொறி போல (அகம். 153/8-9,17-18) காட்சி தந்தன. மாலைப்பொழுதில், பிடாவினது குளிர்ச்சி பொருந்திய மலர்வதற்குரிய கூர்மையான அரும்புகளை வண்டுகள் மலரச் செய்வதால் இடைவிடாது பரவுகின்ற நறுமணத்தைக் கூதிர் காலத்துக்கும், முன்பனிக்காலத்துக்கும் உரிய வாடைக்காற்று பலவிடத்தும் பரப்பியது (அகம். 183/10-13) என்கிறது.

“கரைபாய் வெண்திரை கடுப்ப, பலஉடன்,

நிரைகால் ஒற்றலின், கல்சேர்பு உதிரும்;” (அகம். 199/1-2) 
எனும் பாடல் கரையை வந்து தாக்கும் வெண்ணிற அலையைப் போன்று வரிசையாக வரும் காற்று மோதுவதால், மலையைச் சார்ந்து வளரும் வெண்கடம்பின் முற்றிய வெண்மையான மலர்கள் பலவும் ஒருசேரப் பாறையின் மேல் உதிர்ந்து விளங்கின எனக்குறிப்பிடுகிறது.

அகன்ற வயலிடத்து நீீடு தண்டாக வளர்ந்த கரும்பினது திரண்ட காம்பினையுடைய வெண்ணிறப்பூக்கள், கோடைக்காலத்துப் பூக்கும் பூளைப் பூக்களைப் போல வாடைக்காற்றில் (அகம். 217/3-5) அசைந்தாடியது. செந்நாய்க்கு அஞ்சிய புள்ளிகளையுடைய கலைமான், புள்ளிகள் பொருந்திய அடிமரத்தினைக் கொண்ட விளாமரத்தின் பழத்தின் புன்மையான ஓட்டில் தோன்றிய துளையின்கண், வெப்பத்தோடு வீசுகின்ற மேல் காற்று புகுந்து (அகம். 219/13-16) இசை எழுப்பியது. புதர்கள் பரந்து கிடக்கும் இடத்திலுள்ள அசையும் மூங்கிலிடத்து, வண்டுகள் துளைத்த அழகிய குறுகிய துளைகளின் வழியே மேல்காற்று புகுந்து வருவதால் எழுகின்ற ஓசையானது, நீர் பருகச் செல்லும் பசுக் கூட்டத்திற்குப் பின்னே வரும் நீண்ட கோலினையுடைய ஆயர் கூட்டம் ஊதும் குழல் இசை போன்று இனிமையாக வந்து (அகம். 225/5-8) ஒலித்தது. வாடைக்காற்று எடுத்தலால் உயர்ந்த அழகிய இதழ்களையுடைய செங்காந்தளின் சுருங்கிய அரும்புகள் சுடர் கொண்ட அகல் விளக்குகள் போல விரிந்து (அகம். 235/7-8) மலர்ந்திருந்தன தென்றலானது, பாம்பின் பல்லைப் போன்று அரும்புகள் முதிர்ந்த, மணங்கமழும் கிளைகளையுடைய குராவினை ஊடறுத்து வந்து வீசியது (அகம். 237/3-4) என்கிறது.

மயில் இறகால் தொடுக்கப்பெற்ற மாலையை அணிந்த வீரர்கள், இளைய பசுவினைக் கொன்று அதன் ஊனைத் தின்றனர்; மேல்காற்றானது புழுதியை அளைந்த வண்ணம் ஒலியினை எழுப்பிக் கொண்டு புலால் நாறும் அவ்விடத்தைத் துழாவிச் சென்றது (அகம். 249/12-14) எனவும், நெடிய பெரிய மலையிடத்து குளிர்ச்சி பொருந்திய மேகங்கள் தவழ்ந்து விளையாடும் அருவிகள் வீழ்கின்ற அகன்ற இடத்தில் கடுங்காற்று சுழன்றடித்தது (அகம். 258/5-6) எனவும், அச்சம் தரும் கானகத்தில் கோடைக் காற்றானது கிளைகளில் மோதுவதால் பூனையின் அடியினைப் போன்ற வடிவினைக் கொண்ட குவிந்த அரும்புகளையுடைய இருப்பை மரங்களின் தந்தத்தைக் கடைந்தாற் போன்று காட்சி தரும் வெண்மையான பூக்கள் உதிர்ந்து கீழே சிதறி வீழுந்திருந்தது (அகம். 267/4-7) எனவும் இயம்பப்பட்டுள்ளது.

அருவிநீர் ஒழுகும் அரிய இடத்தில், காட்டு மல்லிகை மலருடன் கூதள மலரையும் சேர்த்துத் தொடுத்த கண்ணியினின்றும் விட்டு நீங்காத மணத்தை அசைந்து வரும் காற்றானது சுமந்து வந்தது (அகம். 272/7-9) எனக்குறிப்பிடுகிறது. மதிலை மறையச் செய்கின்ற பொரிந்த அடியினையுடைய ஆலமரத்தின் ஓன்றாய் தனித்து வீழ்ந்திருக்கும் விழுதினைக் கோடைக் காற்றானது மோதி அசைத்து அவ்வாலமரத்தில் வீற்றிருந்த ஆணும் பெண்ணுமாகிய இரட்டைப் புறாக்களை (அகம். 287/7-9) நீங்கச் செய்தது. வேங்கை மரத்தின் பெரிய கிளைகளில் தழைந்துள்ள ஒளி பொருந்திய தளிர்களில் தூங்கும் பனித்துளிகளை அசைந்து ஆடிவரும் காற்றானது மெதுவாகத் தடவி அவற்றை உதிர்த்ததை (அகம். 298/4-6) தெரிவிக்கிறது. விரைவையுடைய பெருங்காற்றினால் உதிர்க்கப்பட்டுக் காம்பு கழந்த தேக்கின் இலைகள் பலவும் மேலேழுந்து சுழன்று வட்டமிட்டு வானத்து இடமெல்லாம் மறையுமாறு 
பரவிப் பின்னர் மண்ணில் வீ்ந்து அப்பாலை வழிச்செல்வார் செல்லும் வழியறியாது மயங்குமாறு கவர்த்த வழிகளையெல்லாம் மறைத்தது $\quad$ (அகம். 299/5-8) என்பதை அறியமுடிகிறது.

மலையில் நீண்டு வளர்ந்துள்ள செவ்வாழையின் அழகிய இலைகள் அசையும் காற்று அடிக்குந்தோறும் உறங்கும் யானையின் பருத்த உடம்பினை (அகம். 302/1-3) தடவியது. நீர்வற்றிப்போன சுனையில் படிந்து வரும் மேற்காற்றின் தெளிந்த ஒசை, தோலை இழுத்துக் கட்டிய பறையின் ஒலிபோல மலைப் பிளவுகளில் (அகம். 321/2-3) ஒலித்தது. காற்று வீசுதலால் கிளைகளினின்றும் சிச்சிலிப் பறவையின் சிறகுகள் போன்ற மலர்கள், கருமணல் கொண்ட நிலத்தின் மீது உதிர்ந்து வீ்்ந்து (அகம். 324/9-10) அழகு செய்தன.

சுரவழியில் உப்பு வணிகர் வளைந்த நுகத்தடியில் சிவந்த கயிறு கொண்டு பூட்டிய பகடுகள் மணியொலிக்க வருந்தி இழுத்துத் துன்புறும் கொடிய இடங்களில் புழுதியுடன் காற்று (அகம். 329/5-8) சுழன்றடித்தது. வளைந்த அடியினையுடைய பிடா மரத்தின்கண்ணே குளிர்காற்று வீசுதலால் மொட்டுக்கள் மலர்ந்து நிலவொளி போல் காட்சி (அகம். 344/2-3) தந்தது. வெம்மையுடைய கோடைக்காற்றானது செந்நிற மூங்கிலின் அசையும் தண்டினைத் தாக்கி, ஞெம மரத்தின் இலைகளை உதிர்த்தது; அவ்விடத்தே, களர் நிலத்துப் பிறந்த புழுதியை வாரி எடுத்துச் சுழன்று அடித்தது (அகம். 353/7-9) என விளக்கிறது.

"புல்இலை நெல்லிப் புகர்இல் பசுங்காய்

கல்அதர் மருங்கில் கடுவளி உதிர்ப்ப,

பொலம் செய் காசின் பொற்பத் தாஅம்

அத்தம் நண்ணி" (அகம். 363/6-9)

எனும் பாடல் மலைவழியின் பக்கத்தே பெருங்காற்று வீசுதலால் திரண்ட அடிப்பகுதியையும், சிறிய இலையையும் உடைய நெல்லியின் வடுவிலா பசிய காய்கள் உதிர்ந்து பொன்னாலான காசுகள் போலப் பாலைப்பரப்பில் பரவிக்கிடந்தது எனத்தெரிவிக்கிறது.

பெருஞ்செல்வ மிக்க சிறந்து விளங்கும் இல்லத்தில் திருமணம் செய்து கொண்ட மகளிர் தம் கூந்தலைப் போல் மணங்கமழும் வேங்கை மரத்தின் பொன்னிறத் தாதுக்கள் காற்று அடித்தலால் உதிர்ந்து (அகம். 378/1-4) வீழ்ந்தன.

பெரிய கடலின் மிகுந்த ஆழமுடைய பரப்பிடத்தில், காற்றால் செலுத்தப்பட்ட மரக்கலம், நீரை விலக்கி (புறம். 26/1-2) முன்னேறியது. காற்று இயங்கும் திசைகளும், எந்தப்பற்றுதலும் இல்லாது தனித்து நிற்கும் வானமும் ஆகிய இவற்றைச்சென்று அங்கங்கு அளந்தறிந்தவர்களைப் போல நாளும் இவ்வளவின என்று கூறுவோரும் இருந்தனர் (புறம். 30/4-7) என்கிறது. அகன்ற துறையில் பெருங்காற்று திரட்டுவதால் குவிந்ததும் சுவடுகள் பதிவதுமான மணல் மேட்டிலுள்ள மணலின் எண்ணிக்கையினும் பலகாலம் வாழ்வாயாக! (புறம். 55/19-21) என வாழ்த்துகின்றது. பொதுமன்றத்தில் தொங்குகின்ற இழுத்துக் கட்டப்பட்ட முழவினிடத்துக் காற்று மோதிக் கண்ணிடத்துத் தெளிந்த ஓசையை எழுப்புதலைக் கேட்ட அளவில் என் தலைவன் அதியமான் 'அது போர்ப்பறை முழக்கம்' என்று மகிழ்ந்தாக (புறம். 89/7-9) தெரிவிக்கிறது. 
“விரை வளர் கூந்தல் வரை வளி உளர," (புறம். 133/4)

என்ற பாடலின் மூலம் மாட்சிமைப்பட்ட விறலியின் மணம் வளரும் கூந்தலில், மலையின் காற்று வந்தசைந்தது. காற்றின் வேகத்தைப் போன்ற தாவுதலையுடைய குதிரையோடு கொடி அசையும் உச்சியையுடைய தேரினை உடையவர் ஆவார் (புறம். 197/1-2) என்பதை அறியமுடிகிறது. கத்தரிக்கப்பட்ட பிடரிமயிரினை உடைய குதிரைகளை முகந்து செல்வோம் எனின் உடம்பு முழுதும் நிறைந்த வடுவுடனே வீ்ந்து இறந்து, காற்றின் இயக்கம் இன்றிக் கிடக்கும் மரக்கலம்போல, குருதி வெள்ளத்தில் நிறைந்து அவை மிதக்கலாயின (புறம். 368/7-10) என்றும், விரைந்த செலவினையுடைய குதிரைகள் வீசுகின்ற காற்றாக விளங்கின (புறம். 369/7) என்றும் சொல்லப்பட்டுள்ளது.

சங்கால மனிதன் காற்றின் முக்கியத்துவத்தை உணர்ந்து காற்று மண்டலம் மாசுறாத வண்ணம் பாதுகாத்து பராமரித்து வந்ததால் மனிதனுக்கும் மற்ற உயிரினங்களுக்கும் எவ்விதத்திலும் பெரிய பாதிப்புக்கள் ஏற்படவில்லை. இன்று மனிதனிடம் நாகரிகமும் தொழிற்நுட்பமும் மேலோங்கி வருவது பலவிதங்களில் நன்மையளித்து வந்ததாலும் காற்றுமண்டலம் அதிகமாக மாசடையவதற்கு முக்கிய காரணமாக அமைகின்றது. இன்று மனிதர்களும், விலங்குகளும், தாவரங்களும் பல நிலைகளில் பாதிக்கப்படுகின்றன. குறிப்பாக மனிதன் சுவாசிக்க தூயகாற்று கிடைக்காத சூழ்நிலையில் பல்வேறு சுவாச நோய்களுக்கு ஆளாகி மடியக்கூடிய நிலை உருவாகி வருகிறது.

இன்று உலகெங்கினும் புகைமூட்ட பனியால் வருகிறது. ம மனிதன் பல்வேறு நிலைகளில் பாதிப்புக்கு உள்ளாகி வருகின்றான். குறிப்பாக நமது இந்தியத் திருநாட்டில் கடந்த சில மாதங்களுக்கு முன் தலைநகர் டெல்லியில் மக்கள் தூயகாற்று இன்றி தவிர்த்த நிலை ஏற்பட்டது. இதற்கு முக்கிய காரணியாக அமைவது தொழிற்ாலைகளின் புகை, மற்றும் கணக்கற்ற வாகனங்களின் எண்ணிக்கை பெருகியமையும் காரணமாக அமைகின்றன, மேலும் சாலையை விரிவுபடுத்த சாலையோரங்களில் இருந்து மரங்கள் வெட்டப்பட்டதும் முக்கிய காரணமாக அமைகின்றது. மக்கள் தொகை பெருக்கத்தின் காரணமாக உயரமான மாடிவீடுகளில் மக்கள் வசிக்க வேண்டியநிலை காரணமாக போதிய மரம், செடி, கொடிகள் வளர்க்க இயலாமையால் காற்று மாசடைகிறது. பூமியின் அருகிலுள்ள ஆக்சிஜன் வீழ்ச்சிக்கு வழிவகுக்கிறது.

\section{காற்று மாசுபாடு அடைவதைக் கட்டுப்படுத்தும் வழிமுறைகள்}

உலகிலுள்ள அனைத்து உயிரினங்களின் நலமான வளமான வாழ்க்கையைப் பயமுறுத்திக் கொண்டிருக்கும் காற்று மாசுபாட்டின் தீயவிளைவுகளை மக்ள் உணரவேண்டும். தொழிற்சாலைகளின் தயாரிப்புப் பணிகளுக்கான உத்திமுறைகளையும், தொழில் நுணுக்கங்களையும் மாற்றி அமைக்கும்போது மாசுபொருட்கள் காற்றுமண்டலத்தை அடையாமல் தடுக்கலாம் (Xiangdong Li et al., 2019; Sarath K.Guttikunda et al., 2014).

தொழிற்சாலைகளில் பயன்படுத்தப்படும் சில மூலப் பொருட்களில் காற்றை மாசுபடுத்தும் பொருட்களை பயன்படுத்தும் முன்னரே நீக்குவதன் மூலம் சுற்றுப்புறங்களில் மாசுகட்டுப்பாட்டை தவிர்க்கலாம். 
அதிக அளவில் மாசினை ஏற்படுத்தக்கூடிய எரிபொருளுக்குப் பதிலாக இயற்கை எரிவாயுவைப் பயன்படுத்தி மாசினைக் கட்டுப்படுத்தலாம்.

தொழிற்சாலைகளை நகரங்களைவிட்டு தொலைவில் அமைப்பதன் வழியாகவும் நகரங்களில் அதிகமரங்களை வளர்ப்பதன் வழியாக காற்றினை மாசுபடுத்தும் நிலையை தவிர்க்கமுடியும்.

காற்றுமண்டலத்திலுள்ள பல வாயுக்களில் ஓன்று தான் ஓசோன் ஆகும். இந்தப்படலம் பூமியிலுள்ள உயிரினங்களை சூரியனின் ுறற ஊதாக்கதிர்களின் நேரடியான பாதிப்பிலிருந்து பாதுகாக்கும் கவசமாக அமைந்துள்ளது. மனிதனின் செயல்களினால் காற்றுமண்டலத்திலுள்ள இந்த ஓசோன் படலம் அழிந்து வருகிறது. இதனால் பல தீயவிளைவுகள் ஏற்பட்டு வருகிறது. ஒவ்வொரு தனிமனிதனும் பாதுகாப்பதை தனது கடமையாக கொண்டு செயல்படவேண்டும்.

இன்றைய தொழில்நுட்பத்தின் விளைவாக ஏற்படும், காற்றுமாசுபாட்டை தவிர்க்க ஓரே வழி வீடுகளிலும் வளர்ப்பதே நிரந்தரத் தீர்வாகும், இதன் வழியாக மழை வளம் பெருகுவதோடு காற்று தூய்மையடைவதோடு மக்களும், மற்ற உயிரினங்களும் நோய் நொடியின்றி நலமுடன் வளமுடன் வாழ வழியுண்டாகும்.

\section{முடிவுரை}

சங்ககாலத்தில் மர அழிப்பு சிலமுக்கிய காரணங்களுக்காக குறைந்த அளவிலேயே அழிக்கப்பட்டன. தொழிற்சாலைகள் மிகமிகக் குறைந்த அளவிலேயே இருந்தன. இன்று உள்ளது போல மென்மம் (Rubber) வளைமம் (Plastic) போன்ற செயற்கைத்தயாரிப்புகள் அன்று இல்லை எனவே அன்று மக்கள் நோய் நொடியின்றி மகிழ்வோடு வாழ்ந்தனர். இன்று மக்களின் தேவைகள் அதிகமானதால் இயற்கை செயற்கையாக்கப்பட்டு மக்கள் கொடிய நோய்களுக்கு ஆளாகி வருந்தி மனமகிழ்வை இழக்கின்றன.

\section{References}

Alis, A., (Commentator), Pathitrupathu, Third Edition-2007, New Century Book House, Chennai-600098, India.

Balasubramaniyan, K.V., (Commentator), Nattrinai, Third Edition-2007, New Century Book House,Chennai - 600098, India.

Balasubramaniyan, K.V., Viswanathan, A., Nagarajan, V., Thilagavathi, K., (Commentators), Purananuru, Third Edition-2007, New Century Book House, Chennai - 600098, India.

Dhatchenamoorthi, A., (Commentator), Ainkurunuru, Third Edition-2007, New Century Book House,Chennai - 600098, India.

Jeyabal, R., (Commentator), Agananooru, Third Edition-2007, New Century Book House, Chennai 600098, India.

Nagarajan, V., (Commentator), Kurunthogai, Third Edition-2007, New Century Book House, Chennai 600098, India.

Sarath K.Guttikunda, RahulGoel, PallaviPant, Nature of air pollution, emission sources, and management in the Indian cities, Atmospheric Environment, 95 (2014) 501-510. 
Subramaniyan, P., Balasubramaniyan, K.V., (Commentators), Paripadal, Third Edition-2007, New Century Book House, Chennai - 600098, India.

Viswanathan, A., (Commentator), Kalithogai, Third Edition-2007, New Century Book House, Chennai 600098, India.

Xiangdong Li, Ling Jin \& Haidong Kan, Air pollution: a global problem needs local fixes, Nature 570 (2019) 437-439.

Funding: NIL

Acknowledgement: NIL

Conflict of Interest: NIL

\section{About the License:}

\section{(C)}

Attribution 4.0 International (CC BY 4.0)
(C) The author 2020. The text of this article is licensed under a Creative Commons Attribution 4.0 International License 